\title{
Effectiveness of magnesium sulfate as initial treatment of acute severe asthma in children, conducted in a tertiary-level university hospital. A randomized, controlled trial
}

\author{
Silvio Torres ${ }^{a}$ M.D., Nicolás Sticco ${ }^{a}$ M.D., Juan José Bosch ${ }^{a}$ M.D., Tomás Iolster ${ }^{a}$ M.D., \\ Alejandro Siaba $a^{a}$ M.D., Manuel Rocca Rivarola ${ }^{a}$ M.D. and Eduardo Schnitzler ${ }^{a}$ M.D.
}

\begin{abstract}
SUMMARY
Introduction. Magnesium sulfate is a calcium antagonist that inhibits bronchial smooth muscle contraction promoting bronchodilation. It is used for the management of acute severe asthma in children; however most of the studies have been performed in adults.

Objective. To evaluate the effectiveness of intravenous magnesium sulfate for the treatment of pediatric patients with acute severe asthma exacerbations.
\end{abstract}

Population and Methods. A clinical, randomized, controlled trial was conducted between March 2006 and March 2011 at Hospital Universitario Austral. Children with acute severe asthma admitted to the emergency department were randomized into two groups. Group A (control group): standard protocol for the initial treatment of acute asthma exacerbation. Group B: treatment protocol with magnesium sulphate for acute severe asthma exacerbation. The primary outcome was the requirement of invasive or non invasive mechanical ventilation support. Results. One hundred and forty three patients randomized into 2 groups were analyzed. The treatment group included 76 patients receiving magnesium sulfate within the first hour of the initiation of rescue treatment at the hospital, and the control group included 67 patients not treated with magnesium sulphate. Among the patients in the control group, $33 \%(n=22)$ required mechanical ventilation support, compared to only $5 \%(n=4)$ of the patients in the treatment group $(p=0.001)$.

Conclusions. Intravenous infusion of magnesium sulfate during the first hour of hospitalization in patients with acute severe asthma significantly reduced the percentage of children who required mechanical ventilation support.

Key words: magnesium sulphate, acute severe asthma in children, Wood's score, mechanical ventilation support, emergency department.

http:/ /dx.doi.org/10.5546/aap.2012.eng.291

E-mail address:

Silvio Torres:

storres@cas.austral. edu.ar

Conflict of interest:

None.

Received: 12-1-2011 Accepted: 4-26-2012 sympathetic stimulation and prevents acetylcholine release to the axon terminal; therefore promoting a bronchodilating effect. ${ }^{1}$

Magnesium sulphate also has a role in the reduction of inflammation by inhibiting mast cell degranulation and reducing thromboxane, histamine and leukotrienes circulation.

The first publication on the use of magnesium sulphate for the treatment of asthma was the report of a case ${ }^{2}$ sixty years ago, but only in 1989, Skobeloff et al. published the first randomized, double-blind, placebo-controlled trial on the benefits of magnesium sulphate for acute asthma exacerbations. ${ }^{3}$

Several subsequent publications showed that magnesium sulphate was effective for its use both in emergency departments and intensive care units. ${ }^{4,5}$

In the last years, several studies analyzed the effectiveness of magnesium sulphate in acute severe asthma episodes as an intravenous therapy or inhaled together with bronchodilators. However, most studies were developed for adult populations, as shown in the 2000 Cochrane Collaboration systematic review. ${ }^{6}$

In pediatric populations, the study conducted by Ciarallo et al. should be pointed out, with a randomized, double-blind and controlled design based on pulmonary function tests. Among its weakness it is worth mentioning that it only included patients older than 6 years and that pulmonary function tests measurements had been erratic, as shown in the study's editorial discussion. $^{7}$ 
The lack of pediatric studies has been associated with the difficulty of measuring pulmonary function in younger children and the unwillingness of older children to be subjected to a test. For this reason, studies developed in this age range sought to measure the following: an improvement in respiratory scores, hospitalization length-of-stay, and the use or not of mechanical ventilation (MV).

\section{OBJECTIVE}

To evaluate the effectiveness of intravenous magnesium sulphate for severe asthma exacerbations in pediatric patients.

\section{Acronyms:}

$\mathrm{SO}_{4} \mathrm{Mg}$ : Magnesium sulphate.

HUA: Hospital Universitario Austral.

MV: Mechanical ventilation.

PICU: Pediatric Intensive Care Unit.

CPG: Clinical Practice Guidelines.

\section{POPULATION AND METHODS}

Between March 2006 and March 2011, a clinical, open-label, randomized, controlled trial was conducted at Hospital Universitario Austral. The clinical treatment protocol was approved by the Ethics Committee and the Clinical Research Unit of Hospital Universitario Austral. The Clinical Practice Guidelines on acute severe asthma used in this study were prepared by HUA's Clinical Practice Guidelines Sub-Committee and approved by the Ethics Committee.

The study included patients aged 2 to 15 who attended the hospital emergency department due to acute severe asthma, classified according to a Wood's score (Table 1) of 5 or more. This assessment was performed by the Pediatric Intensive Care Unit (PICU) staff doctors, who indicated the patient's admission following such classification (if Wood's score was $\geq 5$ ). Patients with hyperthermia (temperature $\geq 38.3^{\circ} \mathrm{C}\left[100.94^{\circ} \mathrm{F}\right]$ ), systolic blood pressure $<25$ th percentile for their age, treatment with theophylline or aminophylline in the previous 48 hours, and children with a history of kidney, heart or restrictive chronic pulmonary disease were excluded.

Parents or legal guardians were asked to give their written informed consent to participate in the study after understanding the study objectives, scope and likely risks.

Randomization was performed using a random number sequence, a computer and STATA 8.0 software. Two physicians responsible for the study prepared the randomization series, the assignment was then performed using opaque envelopes. A sequence number was assigned to each of these sealed envelopes, thus blinding was ensured. Professionals taking part in the randomization process were different from those in charge of recruitment and data analysis.

A database was built using the following endpoints: age, sex, length-of-stay in PICU, need of $M V$, days of stay in MV, length-of-stay in MV, length-of-stay in intensive care unit, hospitalization length-of-stay, family history of asthma (yes / no), history of at least one admission to the intensive care unit or intermediate care unit (yes/no), background outpatient treatment with bronchodilators, background outpatient treatment with inhaled corticosteroids.

\section{Treatment protocol}

Patients with acute severe asthma seen at the emergency department, whose parents had given their consent for the study, were included and assigned to one of two groups:

- Group A: magnesium sulphate treatment protocol for acute severe asthma exacerbation. (Table 2a)

TABLE 1. Wood's score

\begin{tabular}{|c|c|c|c|}
\hline Endpoints & 0 & 1 & 2 \\
\hline $\mathrm{PaO}_{2}(\mathrm{mmHg})$ & 70-100 en ambient air & $<70$ in ambient air & $<70$ in $40 \% \mathrm{O}_{2}$ \\
\hline Cyanosis & No & In ambient air & In $40 \% \mathrm{O}_{2}$ \\
\hline Inspiratory murmur & Normal & Uneven & Reduced or absente \\
\hline Use of accessories & No & Moderate & Maximum \\
\hline Expiratory wheezes & No & Moderate & Marked \\
\hline Brain function & Normal & Depressed or agitated & Coma \\
\hline
\end{tabular}

A score of $\geq 5$ means a severe attack. 
- Group B: standard initial protocol for acute severe asthma exacerbation. (Table 2b)

All patients were initially treated with 3 doses of inhaled $\beta$-2-adrenergic nebulization (salbuta$\mathrm{mol}$ ) at $0.15 \mathrm{mg} / \mathrm{kg} /$ dose (minimum $2.5 \mathrm{mg}$ and maximum $10 \mathrm{mg}$ every 20 minutes during the first hour), and methylprednisolone at $1 \mathrm{mg} / \mathrm{kg} /$ dose IV. ${ }^{4}$ All patients in the treatment group received IV magnesium sulphate, at $25 \mathrm{mg} / \mathrm{kg}$ (maximum $2 \mathrm{~g}$ ) in a 20-minute infusion, within the first hour after being admitted to the hospital. ${ }^{9}$

A nurse stayed next to the child during the entire infusion time, supervised by the physician who had prescribed the treatment.

The following parameters were monitored on an ongoing basis: heart rate, respiratory rate, blood oxygen saturation, and blood pressure. The pharmacy of Hospital Universitario Austral provided the medication for the study in its usual commercially available presentation for children and adults.

\section{Treatment discontinuation criteria}

The treatment protocol was discontinued and the next stage in the CPGs was initiated if any of the following occurred during the drug infusion:

- Oxygen saturation $<88 \%$ with a mask $\left(\mathrm{FiO}_{2}\right.$ 0.35).

- Marked clinical deterioration evidenced by an increase of $>2$ points in Wood's score from baseline at study enrollment.

- Arterial hypotension $(<25$ th percentile for the patient's age).

- Heart rate $>30 \%$ from baseline at study initiation.

\section{Statistical analysis}

For the sample size required we observed that, in accordance with the reviewed literature, the incidence for positive pressure support is 10 $15 \%{ }^{10,11}$ Taking into consideration a reduction of $20 \%$ of the endpoint "Use of MV" (primary predetermined endpoint) in the treatment group, and assuming a power of $80 \%$ and a $95 \%$ confidence interval (CI), the sample size estimated for the difference in proportion between the 2 groups was 65 patients per arm.

A database was established, where quantitative outcomes were analyzed using median and quartiles, when distribution was not parametric,

TABLE 2. Treatment protocol with IV magnesium sulphate, included in the Clinical Practice Guidelines of Hospital Universitario Austral

TABLE 2A. Treatment group

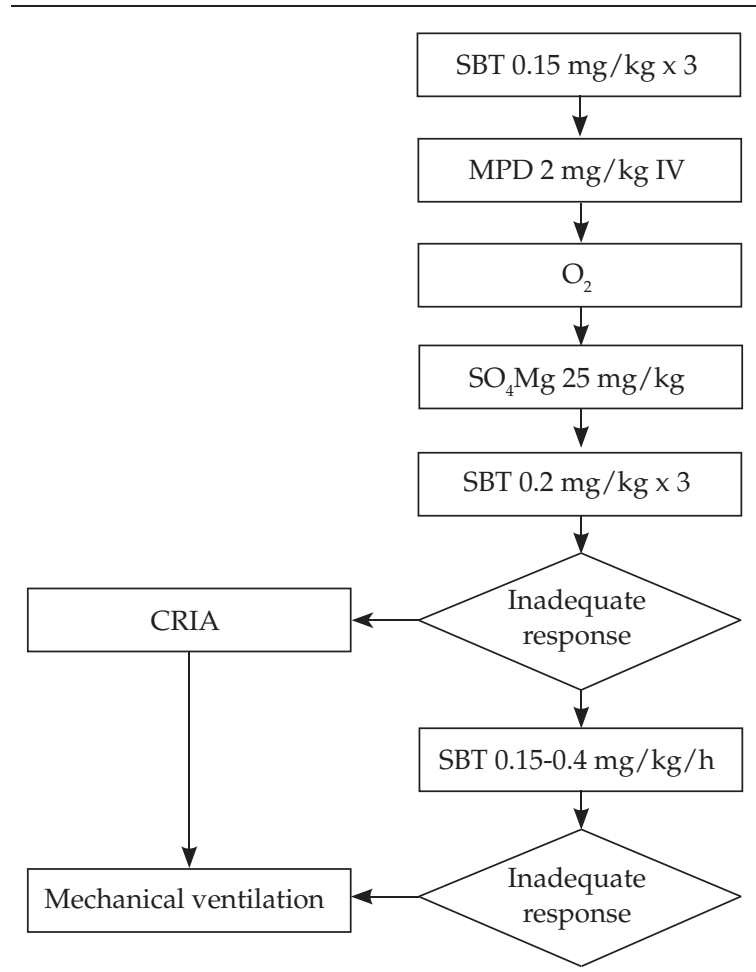

TABLE 2B. Control group

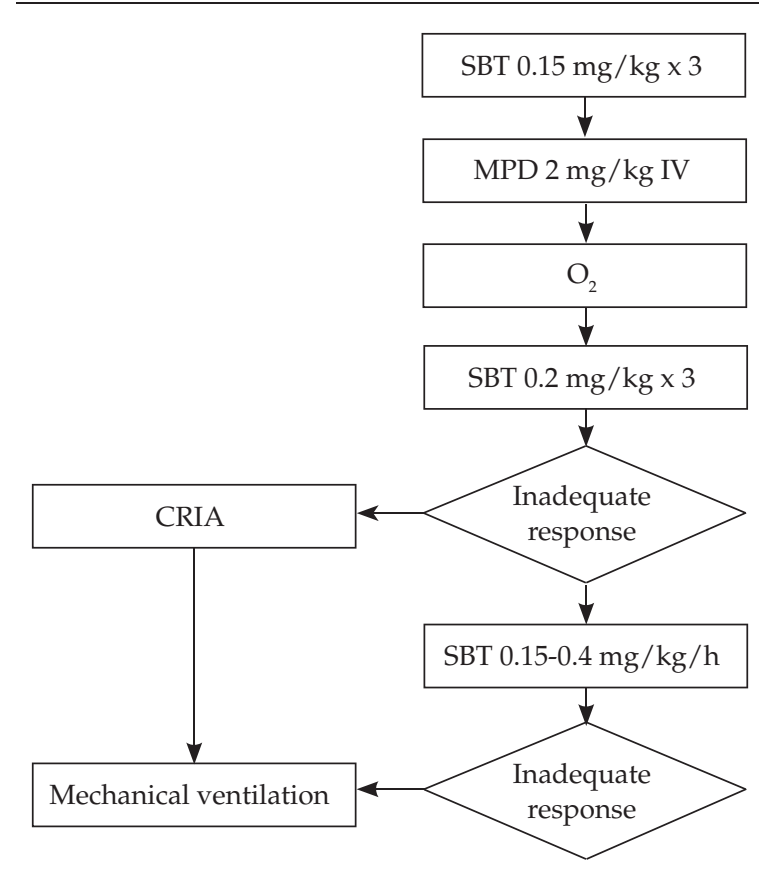

SBT: Salbutamol. MPD: Methyprednisolone. $\mathrm{SO}_{4} \mathrm{Mg}$ : Magnesium sulphate. IARF: Impending Acute Respiratory Failure. 
and using means and standard deviations, when outcomes met alleged normal values. Categorical endpoints were stated in percentages and absolute values.

Continuous endpoints were compared using a t-test (according to their parametric distribution) or a Wilcoxon test, while categorical endpoints were analyzed using a chi-square test $\left(\chi^{2}\right)$ or a Fisher's exact test. A $p$-value of $<0.05$ was considered statistically significant. The statistical analysis was performed on an intention-to-treat basis in both groups.

A multivariate logistic regression analysis was also done. A baseline univariate analysis was done comparing all endpoints with that of the need of mechanical ventilation support; since this was considered influential, the previous treatment with inhaled $\beta$-2-adrenergics and corticosteroids interaction endpoint was established. Those resulting in $p \leq 0.1$ were included in the multivariate analysis. One by one, they were included in the model, analyzing the confounding and modification effect, to eventually reach a predictive model with the following: treatment with magnesium sulphate, family history of asthma, previous treatment with inhaled corticosteroids/ $\beta$-2-adrenergics, and age younger than 60 months.

The statistical software, STATA 8.0 (California, USA) was used.

\section{RESULTS}

During the study period, 148 eligible patients were assisted but parents' consent was not obtained for 5 of them. Therefore, 143 patients diagnosed with acute severe asthma according to Wood's score were randomized. Of these, 76 were included in the treatment group and 67 in the control group. None of the patients met any of the treatment discontinuation criteria.

The baseline characteristics of the individuals included in the groups are shown in Table 3.

Of the patients in the control group, 33\% $(\mathrm{n}=22)$ required MV (6 required invasive $\mathrm{MV}$ and 16 non-invasive MV), compared to only $5 \%(n=4)$ of the patients in the magnesium sulphate group ( 3 required non-invasive MV and 1 invasive MV), with a statistically significant difference $(p=0.001)$. The mean length-of-stay with MV was 5 days (range (r): 2-12) in the control group, and 3 days $(\mathrm{r}: 1-6)$ in the treatment group $(p=0.087)$. The median admission to the PICU was 2 days (r: $1-4)$ in the treatment group, compared to 10 days ( $\mathrm{r}$ : $6-18)$ in the control group $(p=0.0367)$. The median length-of-stay was 7 days in the treatment group (r: 3-12) compared to 19 days in the control group (r: 14-29); this difference was statistically significant $(p=0.046)$. (Table 4$)$

Following the baseline univariate analysis, the endpoints to be included in the multivariate logistic regression analysis were selected according to their statistical significance. The endpoint "history of admission to the PICU", previously considered influential, was eventually not significant in our univariate analysis. It should be mentioned that the confounder of the endpoints "family history of asthma", "outpatient treatment" (inhaled $\beta$ 2 -adrenergics and inhaled corticosteroids as interaction term), and "age younger than 60 months" were finally included in the model together with the endpoint "treatment with magnesium sulphate". This last endpoint had a statistically significant protective effect on the non-use of MV (OR: 0.680, 95\% CI: 0.238-0.836). Among the remaining outcomes analyzed, age younger than 60 months was associated with a statistically significant higher risk of use of MV. (Table 5)

\section{DISCUSSION}

This clinical randomized and controlled trial on the use of magnesium sulphate during the first hour is the first one conducted in this field in Argentina and may serve as the basis for future research on acute severe asthma and its management in the emergency department.

TABle 3. Demographics

\begin{tabular}{lcccc}
\hline & Treatment group $\mathbf{n = 7 6}$ & Control group $\mathbf{n = 6 7}$ & Total & p-value \\
\hline Sex $(\mathrm{M})$ & 33 & 25 & 58 & NS \\
Age in months (median, interquartile range) & $75(30-174)$ & $62(26-168)$ & NS \\
History of admission to the PICU & 27 & 22 & $49 / 143$ & NS \\
Family history & 47 & 59 & $106 / 143$ & NS \\
Outpatient treatment with inhaled $\beta$-2-adrenergics & 28 & 22 & $50 / 143$ & NS \\
Outpatient treatment with inhaled corticosteroids & 61 & 53 & $114 / 143$ & NS \\
Wood's score $(\alpha ;$ SD) & $7.4 ; 2$ & $6.9 ; 1$ & NS \\
\hline
\end{tabular}

$\alpha$ : mean; SD: standard deviation; NS: not significant. 
Our results showed that intravenous magnesium sulphate delivery during the first hour of hospitalization in children with acute severe asthma significantly reduced the requirement of mechanical ventilation support.

In addition, a shorter stay in the PICU and in the hospital was observed in the treatment group (both differences were statistically significant). In the treatment group, the duration of respiratory support was also shorter, but this difference was not statistically significant.

In children younger than 5 years old, a higher risk of MV requirement was observed compared to children older than 5 years old. This difference was statistically significant and independent of the confounders that the other concomitant endpoints of the model may have had. The severity of an acute exacerbation of asthma in young children may make this population susceptible not to improve with the accepted drug treatment and to require a different therapy, such as positive pressure support. In spite of the severity posed by the use of MV, no children died.

In 2005, a metaanalysis of 5 randomized and controlled studies that included 182 children compared magnesium sulphate together with $\beta$ 2 -agonists and intravenous corticosteroids versus placebo. It was concluded that magnesium sulphate provides an additional benefit in acute, moderate and severe asthma by reducing hospitalization in these patients with a decrease of absolute risk by 0.26 (95\% CI $0.12-0.39)$. In four studies a dose of $25 \mathrm{mg} / \mathrm{kg}$ was used, while in the fifth study a dose of $40 \mathrm{mg} / \mathrm{kg}$ was used; no adverse events were observed in any of the studies. ${ }^{8}$
The evidence supporting the use of magnesium sulphate for acute severe asthma has increased over the last years. In 2009, Knut and Halvorsen ${ }^{9}$ endorsed the use of intravenous magnesium sulphate for severe asthma exacerbations in children, after using inhaled $\beta$-2-adrenergics and corticosteroids.

In relation to our study, when patient recruitment started in 2006, there was no sound evidence regarding its use. As of 2009, magnesium sulphate has been recommended in pediatric care. In its 2010 version, the Global Initiative for Asthma (GINA) considers magnesium sulphate level A of evidence in children who do not respond to standard treatment after the first hour of therapy or in those who do not exceed $60 \%$ of the theoretical FEV after an hour of therapy. However, in the different pediatric intensive care units it is not used in a protocolized manner and has not been included in the different clinical practice guidelines. ${ }^{4,5} \mathrm{We}$ believe that our study offers additional evidence by specifically analyzing the use of positive pressure support.

It is widely accepted that both invasive and non-invasive ventilation have deleterious effects on asthma patients, because morbidity and mortality are increased. ${ }^{12}$ However, not all patients with asthma exacerbations will progress to acute respiratory failure, and only $8-24 \%$ of patients are hospitalized in a PICU. ${ }^{13,14}$ In this group of patients, approximately $10-15 \%$ will require $\mathrm{MV} .{ }^{10,11}$

The need for excessive sedation, the controversies regarding ventilation approaches, and the use of positive end expiratory pressure (PEEP) in these patients cast a shadow over the prognosis

TABLE 4. Univariate analysis

\begin{tabular}{lccc}
\hline & Treatment group $\mathbf{n = 7 6}$ & Control group $\mathbf{n = 6 7}$ & $\boldsymbol{p}$-value \\
\hline Need of MV & $5 \%(\mathrm{n}=4)$ & $33 \%(\mathrm{n}=22)$ & 0.001 \\
Length-of-stay in MV (days) $\alpha$ & $3(1-6)$ & $5(2-12)$ & 0.087 \\
Total hospital length-of-stay $\alpha$ & $7(3-12)$ & $19(14-29)$ & 0.046 \\
Length-of-stay in PICU (days) $\alpha$ & $2(1-4)$ & $10(6-18)$ & 0.0376 \\
\hline
\end{tabular}

$\alpha$ : median, interquartile range.

TABLE 5. Logistic regression analysis on the objective clinical improvement in the endpoint called "use of $M V$ "

\begin{tabular}{lcc}
\hline Regression variables & OR (95\% CI) & $p$-value \\
\hline Treatment with magnesium sulphate & $0.680(0.238-0.836)$ & 0.0147 \\
Family history of asthma & $1.239(0.565-3.4103)$ & $(\mathrm{NS})$ \\
Previous treatment with inhaled $\beta$-2-adrenergics and corticosteroids & $1.3669(0.821-5.168)$ & (NS) \\
Age $\leq 60$ months (5 years old) & $2.639(1.205-4.108)$ & 0.041 \\
\hline
\end{tabular}

MV: Mechanical ventilation. 
of these children due to the high risk of complications, such as barotrauma and alveolar air leak syndrome, thus increasing their risk of death. ${ }^{15}$

Currently, the accepted ventilation strategies are based on short inhaling times and prolonged exhaling times, with low tidal volumes, low respiratory rates, pressure-controlled ventilation modes and permissive hypercapnia to avoid barotrauma. ${ }^{9}$

It should be noted that our population comprises a wide age range, from 2 to 15 years old. This is considered a weakness of our study due to the small number of subjects included, which prevents us from conducting a subset analysis, although the age distribution in both groups was balanced.

Another limitation is not having done pulmonary function tests, because it is difficult to implement them in children younger than 6 years old, as pointed out by Ciarallo et al. ${ }^{7}$

The endpoint "use of systemic corticosteroids on an outpatient basis" was not recorded either, which could be important to classify bronchial inflammation status. In relation to the use of Wood's score $\geq 5$ to be included in the study, although it was always done by the same healthcare professionals, it should be noted that subjectivity at assigning a score cannot be avoided.

In spite of an open-label design, those who treated patients were different from those who collected the data, and also different from those who processed and analyzed the data in order to mitigate such bias.

Among the study strengths, homogeneity between both groups regarding their baseline characteristics and severity status to be eligible for the study are worth pointing out, together with the management of confounders by stratification and multivariate regression analysis.

It should be noted that magnesium sulphate is a drug approved by the National Administration of Medicines, Food and Technology of Argentina (ANMAT) for its use in children, as stated in resolution $\mathrm{N}^{\circ}$ 0798. It is safe and available for use, so we believe it is feasible to disseminate its use in the emergency department or PICU as shown by our study and the international literature. ${ }^{16}$

Our findings match the evidence obtained from different reviews in this field, focused on objectives similar to ours, regarding the shorter hospital length-of-stay and the smaller number of children requiring mechanical ventilation support. ${ }^{17}$

\section{CONCLUSIONS}

The intravenous administration of magnesium sulphate during the first hour of the patient's admission to the hospital was associated with a significant decrease in the number of children requiring mechanical ventilation support. ${ }^{17}$

\section{Acknowledgments}

To María Elina Serra M.D., for her collaboration in this study.

\section{BIBLIOGRAPHY}

1. Corbridge TC, Hall JB. The assessment and management of adults with status asthmaticus. Am J RespirCrit Care Med 1995;151(5):1296-316.

2. Hurry V Getal. Blood serum magnesium in bronchial asthma and its treatment by administration of magnesium sulphate. J Lab ClinMed1940;26:340-6.

3. Skobeloff EM, Spivey WH, McNamara RM, Greenspon L. Intravenous magnesium sulphate for the treatment of acute asthma in the emergency department. JAMA 1989; 262:1210-13.

4. NHLBI Guidelines for the Diagnosis and Treatment of asthma. Available at: http.//www.nhlbi.nih.gov/guidelines/asthma.

5. Global strategy for asthma management and prevention 2010 (update). Available at: http./ / www.ginasthma.org.

6. Rowe BH, Bretzlaff JA, Bourdon C, Bota GW, Camargo CA Jr. Magnesium sulphate for treating exacerbations of acute asthma in the emergency department. Cochrane Database Syst Rev 2000;(2):CDO01490.Review.

7. Ciarallo L, Sauer AH, Shannon MW. Intravenous magnesium therapy for moderate to severe pediatric asthma: results of randomized, placebo-controlled trial. J Pediatrics 1996;129:809-14.

8. Cheuk DK, Chau TC, Lee SL. A meta-analysis on intravenous magnesium sulphate for treating acute asthma. Arch Dis Child 2005;90:74-77.

9. Oymar K, Halvorsen T. Emergency presentation and management of acute severe asthma in children. Scandinavian journal of trauma. Res Emerg Med 2009;17:40.

10. Paret G, Kornecki A, Szeinberg A, Vardi A, et al. Severe acute asthma in a community hospital pediatric intensive care unit: a ten-year experience. Ann Allergy Asthma Immunol1998;80:339-44.

11. Beers SI, Abramo TJ, Wiebe RA: Bilevel positive airway pressure in the treatment of status asthmaticus in pediatrics. Am J Emerg Med 2007;25:6-9.

12. Maffei FA, van der Jagt EW, Powers KS, et al. Duration of mechanical ventilation in life-threatening pediatric asthma: description of an acute asphyxial subgroup. Pediatrics 2004;114(3):762-7.

13. Cox RG, Barker GA, Bohn DJ. Efficacy, results, and complications of mechanical ventilation in children with status asthmaticus. PediatrPulmonol1991;11:120-6.

14. Malmstrom K, Kaila M, Korhonen K, et al. Mechanical ventilation in children with severe asthma. PediatrPulmonol2001;31:405-11.

15. Bohn D, Kissoon N. Acute Asthma. Ped Crit Care Med 2001; 2:151-63.

16. Alter HJ, Koepsell TD, Hilty WM. Intravenous magnesium as an adyuvant in acute bronchoespasm: a meta-analysis. Ann Emerg Med 2000;36:191-7.

17. Rodrigo G, Rodrigo C, Burschtin O. Efficacy of magnesium sulphate in acute adult asthma: a meta-analysis of randomized controlled trials. Am J Emerg Med 2000;18:2-18. 